\title{
Effectiveness of Family Stress-Relief Interventions for Patients with Dementia: A Systematic Evaluation of Literature
}

This article was published in the following Dove Press journal: Neuropsychiatric Disease and Treatment

\author{
Dalia Codruța Maria Stoia (D) \\ Adelina Ștefãnuț (iD ${ }^{\prime}$ \\ Romina Moldovan (D) $^{2}$ \\ Lavinia Hogea $\mathbb{D}^{3}$ \\ Cătălina Giurgi-Oncu (1D) ${ }^{4}$ \\ Cristina Bredicean ${ }^{4}$ \\ 'Department of Psychology, West \\ University of Timișoara, Timișoara, \\ Romania; " "Victor Babeș" University of \\ Medicine and Pharmacy, Timișoara, \\ Romania; ${ }^{3}$ Discipline of Psychology, \\ Department of Neuroscience, "Victor \\ Babeș" University of Medicine and \\ Pharmacy, Timișoara, Romania; \\ ${ }^{4}$ Discipline of Psychiatry, Department of \\ Neuroscience, "Victor Babeș" University \\ of Medicine and Pharmacy, Timișoara, \\ Romania
}

Objective: The purpose of this paper is to evaluate the effectiveness of stress-relief interventions for family members of patients with dementia.

Data Source: This analysis includes peer-reviewed articles published between 1989 and 2019, selected from online databases. The introduced keywords were: stress reduction, dementia, or Alzheimer's; program, therapy, intervention, or technique; caregivers.

For the Selection of Studies: We utilized the following inclusion criteria: (1) studies with experimental or quasi-experimental design; (2) study samples that include adult caregivers, who take care of other family members diagnosed with various types of dementia; (3) testing one or more types of psychological inferences presented in the study has been conducted to reduce the stress of patients with dementia; (4) studies written in English and subjected to a peer-review process.

Results: Stress-reduction interventions for patients with dementia appear to have had a statistically significant effect in most of the identified studies.

Conclusion: On a qualitative level, the results show the effectiveness of both pre-test and follow-up interventions, but these results are to be regarded cautiously, considering the heterogeneity of the evaluation tools used and the small number of studies included.

Keywords: stress-relieving interventions, family members, dementia

\section{Introduction}

Globally, in the last decades, the aging process has contributed to a significant increase in the incidence of several degenerative chronic diseases. ${ }^{1}$

Concerning neurodegenerative illness, dementia pathology affects both the patients and their families, causing many severe cognitive, emotional, and relational challenges. ${ }^{2}$ In this context, many studies have investigated the efficacy of stressrelief interventions for the family caregivers of people suffering with dementia. ${ }^{3-6}$

However, existing literature lacks an overview of the effectiveness of these interventions, which would help emphasize the relationship between this disorder in patients and the way it psychologically reflects on their family caregivers. Following, the current paper aims to review the impact of psychological stress-reduction interventions on family caregivers of dementia patients.

Given the high prevalence of stress, there is an increased need for effective methods to reduce it. ${ }^{7}$ Among interventions deemed as effective in reducing negative emotionality and improving the subjective well-being state, practices related to yoga and transcendental meditation have been highlighted, ${ }^{8}$ as well as mindfulness
Correspondence: Cătălina Giurgi-Oncu Department of Neuroscience, "Victor Babeș" University of Medicine and Pharmacy, Timișoara, Romania Email catalina.giurgi@umft.ro 
techniques, physical activation, and change of heart rate variability. ${ }^{9}$ Throughout these practices, the participant develops the ability to focus on the present moment, rather than being captivated by ruminating thoughts and processes. ${ }^{1}$

A potentially promising approach that might help family caregivers in reducing their stress levels is a daycare program for adults. These interventions provide support outside the home, such as socialization, medical care, and therapeutic activities for older adults living with a variety of deficiencies, including dementia. ${ }^{10}$ Gottlieb and Johnson ${ }^{11}$ reported that benefiting from this adult careprogram was associated with a decrease in the perceived stress, anxiety, and somatic disorders in members of the patient's family.

The impact and burden of dementia are significant. Although interventions have been made to reduce patients' stress, the degree of effectiveness is yet to be identified globally. Thus, the present paper aims to analyze the extent to which stress-relief interventions for the family caregivers of dementia patients have been useful. The fact that family caregivers benefit from highly effective interventions can highlight whether the reduction in distress is relevant and whether the effects disturb the caregivers' quality of life. Otherwise, we can focus our attention on alternative psychological variables, that could improve the physical and psychological risks to which they are exposed. At the same time, interventions addressing the difficulties encountered by family caregivers can contribute to maintaining and improving their psychological and physical functioning. ${ }^{12}$ Thus, if caregivers' work is optimal, the risk of them potentially somatizing and developing mental disorders (depression, anxiety) is significantly reduced.

Another important aspect is that the interventions that help maintain the mental and physical health of family caregivers can reduce the cost of their healthcare and contribute to an increase in their quality of life. ${ }^{13}$

Finally, the importance of identifying the effectiveness of these programs is also justified by the fact that the reduction in family caregivers' stress levels can successfully delay patient institutionalization by allowing family members to provide home care for a more extended period. ${ }^{14-16}$ Therefore, as follows, the current paper will explore the extent to which psychological interventions can reduce family caregivers' stress.

\section{Methodology}

This paper presents a systematic search of indexed documents in the following databases: PsychINFO, PubMed, and Ebsco. The search and study process took into account the PRISMA criteria. ${ }^{17}$ Searches were limited to peer-reviewed articles written in English. Given the literature deficiency, we have included studies published in the last 30 years (between 1989 and 2019). The search and abstract search algorithm was, as follows: ("stress reduction") and/or (*"dementia") and/or ("caregivers" or "caretakers" or "caretaker"). We introduced these terms into the search engine of the three databases and saved the results in a bibliographic list.

The cumulative criteria taken into account for a study to be included in this systematic review of the literature were (1) An experimental or quasi-experimental design - specifically, for the experimental studies, the necessary criteria had to include a comparative control group, such as regular treatment, placebo intervention, waiting lists, or absence of treatment. In the case of quasi-experimental design studies, the requirement was the existence of a pre-test assessment demonstrating that there are no significant differences in age, gender, and inter-group education.

(2) Study samples included adult carers, who looked after other family members diagnosed with various types of dementia (more precisely, those who cared for the patients were either spouses, parents, children, or siblings);

(3) Testing one or more types of psychological inferences presented in the study was conducted to reduce the stress of patients with dementia;

(4) The analyses were written in English and underwent a peer-review process.

In the first phase of the study analysis, we only considered research that referred to the realization of a psychological intervention to reduce stress in the title or abstract of the paper. We excluded those with a transversal design, theoretical works, or research (transverse or longitudinal) that referred to "implications for future interventions" without mentioning the existence of a control group or experimental group. At this stage of the analysis process, one team member reviewed all of the articles. At a later stage, when we identified the eligible items, double checks were made for each piece. Following these double-checks, we implemented a data confrontation which consisted of two new members of the team re-checking the identified articles. 
For each eligible item, we collected descriptive data, such as the design type, the size of the control and experimental groups, along with statistical data similar to standard averages and deviations for pre- and post-intervention and follow-up assessments.

\section{Criteria for Inclusion of Articles}

During the analysis of the studies, there were four essential steps: (1) identification, (2) verification, (3) eligibility, and (4) inclusion (see Figure 1 - Prisma Chart). (1) Firstly, we used the same search algorithm for the three databases (PsychINFO, PubMed, Ebsco), from which we identified 60 articles . We only considered researches that mentioned the realization of an intervention, including a control group and an experimental group, in the abstract. Meanwhile, we extensively analyzed the literature on the topic of reducing the stress of patients with dementia, and identified another four significant reviews for our research. The additional performed search consisted of introducing key terms on Google Academic, which resulted in 4 articles being identified. (2) The second step was to remove duplicate articles, which limited the database to 47 items that matched our theme, in terms of the abstract. Following this, we eliminated four papers: two that did not meet the initial criteria, and two more that were not available as full-text versions (the authors were contacted, but we did not receive a response from them). (3) The third step consisted of the detailed analysis of the eligibility of all articles, which resulted in 35 items having to be excluded, as they either presented non-experimental studies, included caregivers that were not family members, or failed to measure the stress variable. (4) The final statistical
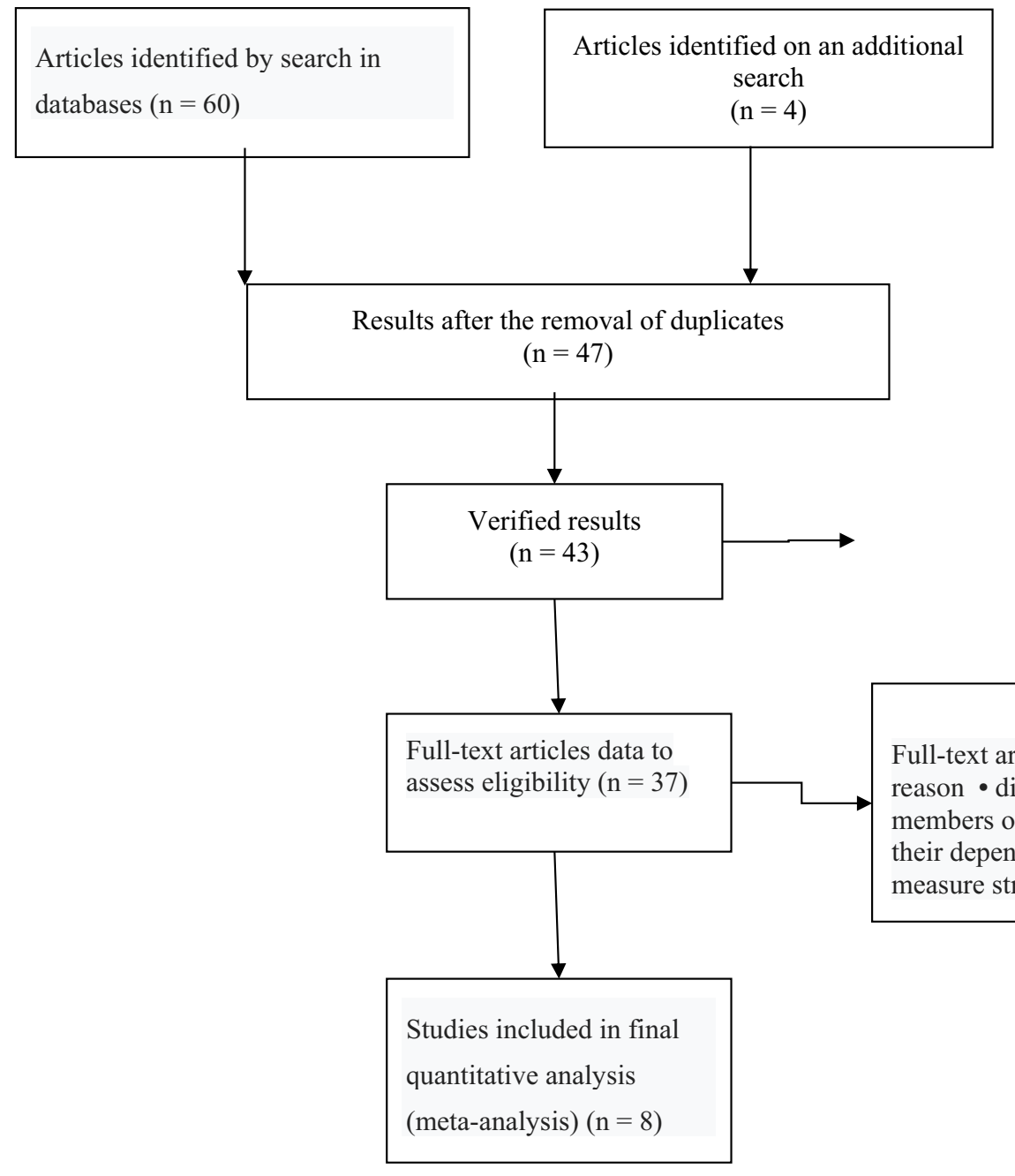

Excluded articles

- non-experimental studies $(\mathrm{n}=6)$
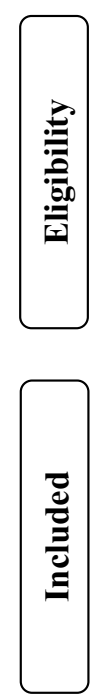

Full-text articles excluded with reason - did not include family members or did not only include their dependents or did not measure stress $(\mathrm{n}=29)$

Figure I Prism chart - The process of determining the eligibility of articles. Copyright @2009 prisma. adapted from liberati a, altman dg, tetzlaff $\mathrm{j}$, et al. the prisma statement for reporting systematic reviews and meta-analyses of studies that evaluate health care interventions: explanation and elaboration. plos med. 2009;6(7):el000100. doi: $10.1371 /$ journal.pmed. $1000100 .^{17}$ 


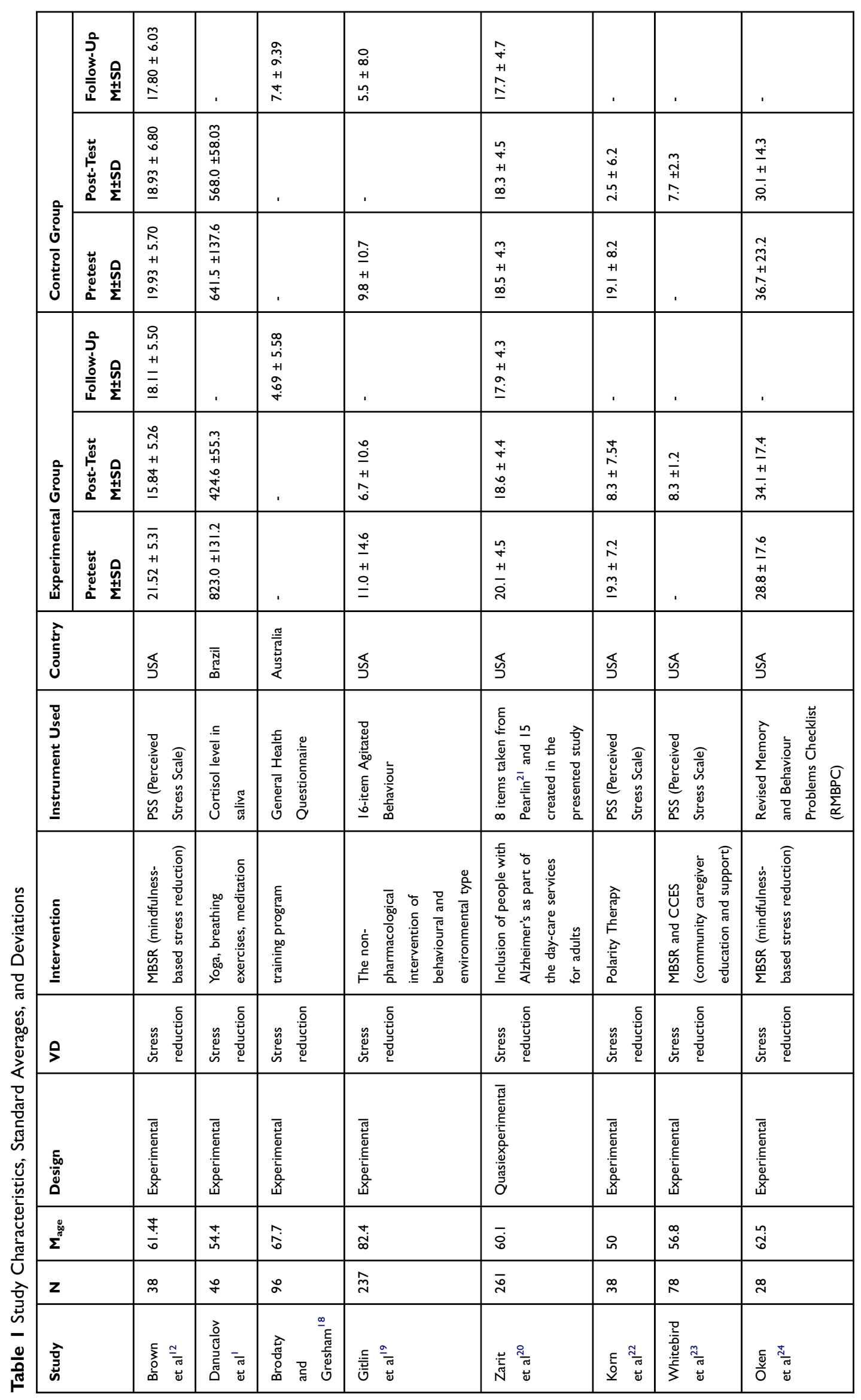


analysis was carried out on the eight identified eligible items (dated between 1989 and 2019).

\section{Data Extraction and Study Encoding}

For the eight eligible articles, we extracted the information considered relevant to the brief description of the methodology: design type, number of participants, averages, type of intervention, the instrument used (see Table 1 - Study characteristics, standard averages, and deviations). There is a risk of bias in the results, due to the small number of studies included and the different measurement instruments used in the studies included.

\section{Results}

Following the systematic review of the literature, we found 60 studies that looked at the impact of the effectiveness of interventions aimed at reducing the stress of family caregivers with arelative diagnosed with Alzheimer's disease. Of these, following the elimination of duplicates, 47 studies were analyzed. Double trials conducted by the team revealed 39 reviews that did not meet the established criteria, either by representing non-experimental studies or by not targeting family members as beneficiaries of stressrelief interventions. Thus, eight studies were included in this literature review (7 experimental studies and 1 quasiexperimental study), with a total of 822 participants.

Considering the heterogeneity of the evaluation tools used and the small number of studies identified, we found that it would be unsuitable to use a statistical analysis characteristic of meta-analytical studies or any other form of quantitative analysis. Table 1 (see Table 1 - Study characteristics, standard averages, and deviations) provides an overview of the impact of stress-reduction interventions on family caregivers of Alzheimer's patients.

\section{Discussions and Conclusions}

In this paper, we attempted to create an overview of the effectiveness of stress-reduction interventions on family caregivers of dementia patients, focusing on two crucial moments: the immediate and the delayed effect, following the intervention. As mentioned in the introduction, there are many chronic degenerative diseases, ${ }^{1}$ with one of the most frequent of the neurodegenerative pathologies being dementia. This disease affects both the patient, but also the family, or caregivers. Capistrant et $\mathrm{al}^{25}$ mention that caregivers are equally exposed to the adverse effects that distress can generate, such as low quality of life, physical and psychological risks (social isolation, burnout, sleep disorders, depression, and various others). Specialty literature includes studies aimed at stressrelief interventions for family caregivers of patients with dementia. $^{3-6}$ However, their degree of effectiveness is not known globally. Therefore, there are no meta-analytical studies that show the overall extent to which these various stressreduction interventions are useful for patients with dementia.

This review of the literature has analyzed a series of 60 scientific articles, of which only eight have met the inclusion criteria. However, this paper has provided enough data to continue conducting a systematic literature review.

Evidence in the scientific publications, so far, suggests that the long-term benefit of stress-relief interventions for family caregivers of people living with dementia is limited. ${ }^{23}$ The results of a systematic literature analysis can be the first argument in favor of the effectiveness of these interventions. They can gain specialists' attention regarding the importance of developing psychosocial programs for people living with Alzheimer's. In coordination with current literature, this work can not outline or, even less so, fully demonstrate, the effectiveness of programs to reduce the stress of families of dementia patients; however, it manages to highlight the significant results obtained in the studies described. ${ }^{12,26-28}$

\section{Practical Implications}

The caregiver-patient relationship is hugely relevant in disease progression, which makes the impact of stressors affect both of them alike. If the discomfort associated with the care of a patient with dementia is kept under control, through interventions to reduce it or to deal with situations with a negative emotional impact, mental disorders may be avoided. Also, if the family provides appropriate support to the patient, this can help improve their overall health.

From a practical point of view, this work is particularly relevant for the field of clinical psychology, as clinical therapists and psychologists may consider that the effect of stress-relief interventions on patients with dementia may be statistically significant with a practical approach, especially over time. Thus, our results cannot ensure the effectiveness of stress-reduction interventions, nor do they confirm their inefficacy, which means that mental health practitioners may consider the implementation of these types of interventions and can, subsequently, monitor their impact at the individual level. As mentioned previously, this neurodegenerative disease also affects family members or caregivers, thus further highlighting the importance that these interventions have. 


\section{Limitations and Future Directions}

The small number of studies included in the analysis (less than 10) represents the main limitation of this work. Additionally, the heterogeneity of the evaluation tools that were employed urged us to limit this to a systematic analysis of the literature. We acknowledge that we have not found enough studies that adequately address this issue, potentially because their implementation requires a longer time, as well as valuable quantitative and qualitative resources. The clinical literature is quite arid, making it challenging to control complex variables. Although the search algorithm that we used has dramatically reduced the identified study palette, for example, by using only the term "stress reduction", we have chosen to strictly maintain the initially proposed eligibility criteria, in order to increase the accuracy of statistical results.

Because the implications of the caregiverpatient relationship have been established, for future directions, we would suggest a more complex meta-analytical approach of stress-relieving interventions aimed at family caregivers or physicians. Meta-analytical strategies are elaborate, but offer a complete picture of clinical reality. We also suggest that future studies could look at the effectiveness of stress-relieving interventions that take into account the phase of illness (onset or terminal phase) in terms of the burden for family members.

Also, because most of the studies were conducted in the USA, the results of the present analysis have a low degree of generalizability for other populations. Given that the World Health Organization ${ }^{29}$ considers dementia a public health issue, with $60 \%$ of all people suffering with dementia currently living in low- and middle-income countries, and with the expectation of most new cases $(71 \%)$ to originate in those countries, the development of interventions that take into account the decrease in stress levels of family caregivers of dementia patients can represent a future research direction of interest in other countries, outside the USA.

The results that were obtained in this study may also be useful in the case of other progressive diseases, where the patient requires the practical care and support of their family. An example of such a disease is cancer, for which the literature highlights the wide array of psychological consequences that the family caregivers must face. $^{30,31}$ Thus, the application of interventions that have been proven effective in reducing the stress of the family caregivers of dementia patients may constitute a new direction in the research involving family caregivers of oncological patients .
Moreover, through the purpose of identifying ways to create social and physical environments that promote good health for all, special attention is granted to care providers. Often called the invisible second patients, caregivers need accessible and effective interventions tailored to their needs. Because of the lack of time, geographical barriers and social stigma are often invoked by caregivers when refusing to participate in interventions; therefore the development of programs that overcome these drawbacks would be welcome. In this sense, the design of interventions that use the telephone, the internet, or video conferencing as means of communication, may be useful.

Finally, caregiver burden is associated with increased institutionalization of patients with dementia. ${ }^{32}$ However, while nursing home placement helps to reduce the direct care obligations on family caregivers, it does not necessarily reduce caregiver distress. Relief and reduced stress may follow institutionalization ${ }^{33}$, but so can guilt anger, anxiety, depression, and financial problems. ${ }^{34}$ Therefore, continuing the development of interventions to reduce the stress of family caregivers of patients with dementia can contribute both to preserving their mental well-being and reducing the use of medical resources.

\section{Disclosure}

The authors report no conflicts of interest in this work.

\section{References}

1. Danucalov MAD, Kozasa EH, Ribas KT, et al. A yoga and compassion meditation program reduces stress in familial caregivers of Alzheimer's disease patients. Evid Based Complement Altern Med. 2013.

2. Savla J, Roberto KA, Blieszner R, Cox M, Gwazdauskas F. Effects of daily stressors on the psychological and biological well-being of spouses of persons with mild cognitive impairment. J Gerontol Ser B. 2011;66(6):653-664. doi:10.1093/geronb/gbr041

3. Akkerman RL, Ostwald SK. Reducing anxiety in Alzheimer's disease family caregivers: the effectiveness of a nine-week cognitivebehavioral intervention. Am J Alzheimer's Dis Other Dementias. 2004;19(2):117-123. doi:10.1177/153331750401900202

4. Mittelman MS, Roth DL, Coon DW, Haley WE. Sustained benefit of supportive intervention for depressive symptoms in caregivers of patients with Alzheimer's disease. Am $J$ Psychiatry. 2004;161 (5):850-856. doi:10.1176/appi.ajp.161.5.850

5. Belle SH, Burgio L, Burns R, et al. Providing support to dementia caregivers improve their quality of life. Ann Intern Med. 2006;145 (10):I39.

6. Elliott AF, Burgio LD, DeCoster J. Enhancing caregiver health: findings from the resources for enhancing Alzheimer's caregiver health II intervention. J Am Geriatr Soc. 2010;58(1):30-37. doi:10.1111/j.15325415.2009.02631.x

7. Henriques G, Keffer S, Abrahamson C, Horst SJ. Exploring the effectiveness of a computer-based heart rate variability biofeedback program in reducing anxiety in college students. Appl Psychophysiol Biofeedback. 2011;36(2):101-112. doi:10.1007/s10484-011-9151-4 
8. Yoshimura M, Kurokawa E, Noda T, et al. Disaster relief for the Japanese Earthquake-Tsunami of 2011: stress reduction through the transcendental meditation technique. Psychol Rep. 2015;117 (1):206-216. doi:10.2466/02.13.PR0.117c11z6

9. Van der Zwan JE, de Vente W, Huizink AC, et al. Physical activity, mindfulness meditation, or heart rate variability biofeedback for stress reduction: a randomized controlled trial. Appl Psychophysiol Biofeedback. 2015;40(4):257-268. doi:10.1007/s10484-015-9293-x

10. Weissert WG. Adult Day Care: Findings from a National Survey. Johns Hopkins University Press; 1990.

11. Gottlieb BH, Johnson J. Impact of Day Programs on Family Caregivers of Persons with Dementia. Gerontology Research Centre \& Psychology Department, University of Guelph; 1995.

12. Brown KW, Coogle CL, Wegelin J. A pilot randomized controlled trial of mindfulness-based stress reduction for caregivers of family members with dementia. Aging Ment Health. 2016;20(11):1157-1166. doi:10.1080/13607863.2015.1065790

13. Mittelman M. Taking care of the caregivers. Curr Opin Psychiatry. 2005;18(6):633-639. doi:10.1097/01.yco.0000184416.21458.40

14. Gaugler JE, Edwards AB, Femia EE, et al. Predictors of institutionalization of cognitively impaired elders family help and the timing of placement. J Gerontol Ser B. 2000;55(4):P247-P255. doi:10.1093/ geronb/55.4.P247

15. Mausbach B, Coon DW, Depp C, et al. Ethnicity and time to institutionalization of dementia patients: a comparison of Latina and Caucasian female family caregivers. J Am Geriatr Soc. 2004;52 (7):1077-1084. doi:10.1111/jgs.2004.52.issue-7

16. Stevens A, Owen J, Roth D, et al. Predictors of time to nursing home placement in White and African American individuals with dementia J Aging Health. 2004;16(3):375-397. doi:10.1177/0898264304264206

17. Liberati A, Altman DG, Tetzlaff J, et al. The PRISMA statement for reporting systematic reviews and meta-analyses of studies that evaluate health care interventions: explanation and elaboration. PLoS Med. 2009;6(7):e1000100. doi:10.1371/journal.pmed.1000100

18. Brodaty H, Gresham M. Effect of a training programme to reduce stress in carers of patients with dementia. BMJ. 1989;299 (6712):1375-1379. doi:10.1136/bmj.299.6712.1375

19. Gitlin LN, Winter L, Dennis MP, et al. A biobehavioral home-based intervention and the well-being of patients with dementia and their caregivers: the COPE randomized trial. JAMA. 2010;304(9):983-991. doi:10.1001/jama.2010.1253

20. Zarit SH, Stephens MAP, Townsend A, Greene R. Stress reduction for family caregivers: effects of adult day care use. J Gerontol Ser B. 1998;53(5):S267-S277. doi:10.1093/geronb/53B.5.S267

21. Pearlin LI, Mullan JT, Semple SJ, Skaff MM. Caregiving and the stress process: an overview of concepts and their measures. Gerontologist. 1990;30:583-594.
22. Korn L, Logsdon RG, Polissar NL, et al. A randomized trial of a CAM therapy for stress reduction in American Indian and Alaskan native family caregivers. Gerontologist. 2009 ;gnp032.

23. Whitebird RR, Kreitzer M, Crain AL, et al. stress reduction for family caregivers: a randomized controlled trial. Gerontologist. 2013;53(4):676-686. doi:10.1093/geront/gns126

24. Oken BS, Fonareva I, Haas M, et al. Pilot controlled trial of mindfulness meditation and education for dementia caregivers. J Alter Complement Med. 2010;16(10):1031-1038. doi:10.1089/acm.2009.0733

25. Capistrant BD, Moon JR, Berkman LF, Glymour MM. Current and long-term spousal caregiving and onset of cardiovascular disease. J Epidemiol Community Health. 2011;66(10):951-956.

26. Bourgeois MS, Schulz R, Burgio L. Interventions for caregivers of patients with Alzheimer's disease: a review and analysis of content, process, and outcomes. Int J Aging Human Devel. 1996;43(1):35-92. doi:10.2190/AN6L-6QBQ-76G0-0N9A

27. Gottlieb BH, Wolfe J. Coping with family caregiving to persons with dementia: a critical review. Aging Ment Health. 2002;6(4):325-342. doi:10.1080/1360786021000006947

28. Schulz R, Martire LM, Klinger JN. Evidence-based caregiver interventions in geriatric psychiatry. Psychiatric Clin North Am. 2005;28 (4):1007-1038. doi:10.1016/j.psc.2005.09.003

29. World Health Organization. EUR/RC66/6 Add.1: document on development of draft global action plan on public health response to dementia for regional committees. Available from: http://www.euro. who.int/_data/assets/pdf_file/0020/316145/66wd06e_Add.1 Dementia_160630.pdf. Accessed December 12, 2019.

30. Williams AL, McCorkle R. Cancer family caregivers during the palliative, hospice, and bereavement phases: a review of the descriptive psychosocial literature. Palliative Supportive Care. 2011;9 (03):315-325. doi:10.1017/S1478951511000265

31. Li QP, Mak YW, Loke AY. Spouses' experience of caregiving for cancer patients: a literature review. Int Nurs Rev. 2012;60 (2):178-187. doi:10.1111/inr.12000

32. Spitznagel MB, Tremont G, Davis J, Foster SM. Psychosocial predictors of dementia caregiver desire to institutionalize: caregiver, care recipient, and family relationship factors. J Geriatr Psychiatry Neurol. 2006;19(1):16-20. doi:10.1177/0891988705284713

33. Brodaty H, Hadzi-Pavlovic D. Psychosocial effects on carers of living with persons with dementia. Aust $N Z J$ Psychiatry. 1990;24:351-361. doi:10.3109/00048679009077702

34. Schulz R, O’Brien A, Czaja S, et al. Dementia caregiver intervention research in search of clinical significance. Gerontologist. 2002;42 (5):589-602. doi:10.1093/geront/42.5.589
Neuropsychiatric Disease and Treatment

\section{Publish your work in this journal}

Neuropsychiatric Disease and Treatment is an international, peerreviewed journal of clinical therapeutics and pharmacology focusing on concise rapid reporting of clinical or pre-clinical studies on a range of neuropsychiatric and neurological disorders. This journal is indexed on PubMed Central, the 'PsycINFO' database and CAS, an is the official journal of The International Neuropsychiatric Association (INA). The manuscript management system is completely online and includes a very quick and fair peer-review system, which is all easy to use. Visit http://www.dovepress.com/testimonials.php to read real quotes from published authors. 